\title{
Some Examples of Empirical Fourier Analysis in Scientific Problems
}

DAVID R. BRILLINGER University of California, Berkeley, California

"One can FT anything-often meaningfully."

J. W. Tukey

\section{INTRODUCTION}

As a concept and as a tool, the Fourier transform is pervasive in applied mathematics, computing, mathematics, probability and statistics as well as in substantive sciences such as chemistry, geophysics and physics. This chapter presents a review of such applications and then four personal analyses of scientific data based on Fourier transforms. Specific points made include: Fourier analysis is conceptually simple, its concepts often have direct physical interpretations, useful statistical properties are available, and there are various interesting connections between the mathematical and physical concepts.

By Fourier analysis is meant the study of spaces and functions, making substantial use of sine and cosine functions. The subject has a long and glorious history. In particular, fundamental work has been carried out by both mathematicians and applied scientists. Fourier analysis remains of interest to mathematicians because generalizations seem inexhaustible and because there are continual surprises. Classic works by mathematicians 
include: Wiener (1933), Bochner (1959, 1960) and Zygmund (1968). These particular authors are concerned with functions on the line or on a general Euclidian space. Works on extensions to general groups include: Loomis (1953), Rudin (1962), Hewitt and Ross (1963), Katznelson (1976). More recent books are Terras (1988) and Körner (1989), the former particularly addressing the nonabelian case, the latter presenting a variety of historical examples and essays on specific topics.

In contrast, the Fourier transform is of interest to statisticians because it proves inordinately useful in the analysis of data and eases the development of various theoretical results. Noteworthy contributions to statistics have been made by Slutsky (1934), Cramér (1942), Good (1958), Yaglom (1961), Tukey (1963), Hannan (1965, 1966), Priestley (1965), Bloomfield (1976), Diaconnis $(1988,1989)$. Slutsky developed some of the statistical properties of the Fourier transform of a stretch of time series values. Cramér set down a Fourier representation (see Sec. 4.1) for stationary processes. The representation admitted many extensions and made transparent the effect of a variety of operations on processes. Good and Tukey indicated how the transform could be computed recursively and hence quickly in certain circumstances. Yaglom extended the domain of application to processes defined on compact and locally compact groups. Hannan considered problems for other groups than Yaglom and presented material relevant to practical applications. Priestley provided a frequency domain representation to describe nonstationary processes. Bloomfield made complicated results available to a broad audience. Diaconnis considered symmetric and permutation groups and the analysis of ordered data.

Particular uses of the empirical Fourier transform include: the development of estimates of finite dimensional parameters appearing in time series models (Whittle (1952), Dzhaparidze (1986), Feuerverger (1990)), the assessment of goodness of fit of models (Feigin and Heathcote (1976)), and the deconvolution of random measurements (Fan (1992)). Fourier analysis has a special place amongst the tools of statistics for the concepts often have their own physical existence.

There are special computational, mathematical and statistical properties and surprises associated with the Fourier transform. These include: the central limit theorems for the stationary case with approximate independence at particular frequencies, the existence of fast Fourier transforms, (Good (1958), Tukey (1963), Cooley and Tukey (1965), Rockmore (1990)) the need for convergence factors, the ideas of aliasing.

Section 2 concerns some particular physical situations. Section 3 contains pertinent background from analysis. Section 4 contains stochastic background. Section 5 presents analyses of four data sets from the natural sciences and the author's experience. The examples highlight 
approximation, shrinkage estimation, the method of stationary phase, central limit theorems and uncertainty estimation. The first example, concerning crystalographic data, involves the empirical representation of a basic function on the plane by an expansion in sines and cosines. This makes sense because of periodicities inherent in the crystal structure. The example also involves shrinkage of the coefficients of the expansion in order to obtain improved estimates. The second analysis is of a record of an earthquake that took place in Siberia as recorded at Uppsala, Sweden. The oscillatory character of the data may be understood heuristically via the method of stationary phase, to be described below. A model of the transmission medium is constructed and model assessment carried out by a sliding or dynamic Fourier analysis. This last may be viewed as a form of wavelet analysis. The third analysis, concerned with nuclear magnetic resonance (NMR) spectroscopy, employs Fourier analysis to obtain physical insight into the behavior of an input-output system, and then makes use of cross-spectral analysis to estimate the transfer function of the system. The periodogram of the residuals is employed to assess the fit. The final example involves both wavelet and Fourier analysis. It is concerned with the question of whether a microtubule moves steadily or via jumps. The Fourier analysis is employed in this case to obtain uncertainty estimates in the presence of stationary noise. Section 6 contains conclusions and indicates open problems.

\section{SOME PHYSICAL EXAMPLES OF FOURIER ANALYSIS}

Cycles, periods, and resonances have long been noted in scientific discussions of astronomy, vibrations, oceanography, sound, light and crystalography amongst other fields. In technology oscillations occur often for example in telephone, radio, TV and laser engineering. Natural operations occur commonly that correspond with linear and time invariant systems as defined in Section 3 below. These are the eigenoperations of Fourier analysis.

Fourier analysis is sometimes tied specifically to the physics of a problem. For example Bazin et al. (1986) physically demonstrate the operations/ concepts of translation, linearity, similarity, convolution and Parseval's theorem for the Fourier transform via diffraction experiments with laser light. The Fourier transform here is formed via a lens. See Goodman (1968) Shankar et al. (1982), Glaeser (1985) for a discussion of the optics involved.

An important example arises in radio astronomy. Suppose there is an array of receivers. Suppose there is a small incoherent source, at great distance, producing a plane travelling wave. If $Y(x, y, t)$ denotes the radio field measurement made at time $t$ on a telescope located at position $(x, y)$, then 


$$
E\{Y(x+u, y+\nu, t) \overline{Y(x, y, t)}\}=\iint f(\alpha, \beta) e^{i(u \alpha+\nu \beta)} d \alpha d \beta
$$

where $(\alpha, \beta)$ are the coordinates of the source of interest in the sky and $f(\alpha, \beta)$ is its brightness distribution as a function of $(\alpha, \beta)$. In other words, the Fourier transform is the quantity observed. The result Eq. (2.1) is known as the van Cittert-Zernike Theorem, see Born and Wolf (1964).

Linear time invariant systems abound in nature. They have the property of carrying cosinusoids into cosinusoids. Nowadays in science there is much concern with nonlinear operations and phenomena. Impressively, the classic trigonometric identity

$$
[\cos \lambda t]^{2}=\frac{1}{2} \cos 2 \lambda t+\frac{1}{2}
$$

is "demonstrated" in Yariv (1975) via a color plate showing red laser light becoming blue on passing through a crystal. The crystal involved squares the signal as in Eq. (2.2). A wavelength of $6940 \AA$ (red) becomes one of 3970 A (blue). Bloembergen (1982), Moloney and Newell (1989) discuss such nonlinear aspects of light. The appearance of harmonics such as in Eq. (2.2) leads to a consideration of higher-order spectra.

The Fourier transform is continually employed in the solution of equations of motion associated with physical phenomena and mathematicians have focussed on consequent cycles and harmonics. For example, Hirsch (1984) has remarked that "Dynamicists have always been fascinated (not to say obsessed) by periodicity." In that connection Ruelle (1989) makes effective use of the Fourier transform in the study of dynamic systems, specifically addressing aspects of chaos, periods and scaling.

The Fourier transform leads to entities with direct physical interpretations. One can point to a variety of success stories of the application of Fourier analysis. Michaelson (1891a,b) measured visibility curves, essentially the modulus of a Fourier transform, and after an inversion thereby inferred that the red hydrogen line was a doublet. This inference of splitting ultimately led to important developments in quantum mechanics. Tidal components caused by the sun, moon and planets have been isolated by Fourier analysis, see Cartwright (1982), Båth (1974), Bracewell (1989). Katz and Miledi (1971) inferred the mechanism of acetylcholine release via a Fourier analysis. Bolt et al. (1982) saw a fault rupturing in an earthquake by a frequency-wavenumber spectral analysis. Finally it may be noted that R. R. Ernst received the 1991 Nobel Prize in Chemistry for developing the technique of Fourier transform spectroscopy, see Amato (1991). A discussion of a variety of other physical examples may be found in Lanczos (1966), Båth (1974), Bracewell (1989). 


\section{SOME ANALYTIC BACKGROUND}

\subsection{The Fourier Case}

Consider a square integrable function $g(x), 0 \leq x<2 \pi$. In this simple case Fourier analysis is built upon the values

$$
c_{k}=\frac{1}{2 \pi} \int_{0}^{2 \pi} e^{-i k x} g(x) d x
$$

$k=0, \pm 1, \pm 2, \ldots$, and Fourier synthesis on expansions

$$
g(x) \approx \sum_{k=-\infty}^{\infty} c_{k} e^{i x k}
$$

The functions $\exp \{i k x\}, k=0, \pm 1, \pm 2, \ldots$ here are orthogonal on $[0,2 \pi)$ and this connects Eqns. (3.1) and (3.2).

One important use of Fourier methods is the approximation of functions. If the values $c_{k}, k=0, \pm 1, \ldots \pm K$ of Eq. (3.1) are available, a naive approximation to $g(x)$ is provided by

$$
\sum_{k=-K}^{K} c_{k} e^{i x k}
$$

However early researchers found that the approximation of Eq. (3.3) was often improved by inserting multipliers, $w_{k}^{K}$, such as $1-|k| / K$, into the expansion and employing

$$
g^{K}(x)=\sum_{k=-K}^{K} w_{k}^{K} c_{k} e^{i x k} .
$$

instead of Eq. (3.3). Defining the kernel

$$
W^{K}(x)=\sum_{k=-K}^{K} w_{k}^{K} e^{i x k}
$$

Eq. (3.4) can be written

$$
\int_{0}^{2 \pi} W^{K}(y-x) g(x) d x
$$

and one sees that Eq. (3.4) is a weighted average of the desired $g(\cdot)$. The effect of the multipliers, in some cases, is to improve the approximation by damping down the more rapidly oscillating terms in the expansion. This idea of damping down will recur below in the consideration of shrinking to improve estimates. The expression of Eq. (3.5) may be used to study directly the effect of the kernel function on the approximation. Timan (1963), Butzer 
and Nessel (1971) are books specifically concerned with approximations based on Fourier expressions.

In work with data values $Y_{t}$ observed at $t=0, \ldots, T-1$ one might replace Eq. (3.1) with

$$
\frac{1}{T} \sum_{t=0}^{T-1} \exp \left\{\frac{-i 2 \pi k t}{T}\right\} Y_{t}
$$

having written $g(2 \pi t / T)=Y_{t}$. As referred to earlier there are fast algorithms to evaluate this.

A second important use of Fourier analysis is in the study of time invariant systems. A simple linear time invariant system is described by

$$
Y_{t}=\sum_{t=-\infty}^{\infty} c_{t-s} X_{s}
$$

i.e., a convolution. The response of this system to the input $X_{t}=\exp \{i \lambda t\}$ is

$$
Y_{t}=C(\lambda) X_{t}
$$

with $C(\lambda)$ the Fourier transform

$$
C(\lambda)=\sum_{s=-\infty}^{\infty} e^{-i \lambda s} c_{s}
$$

for $0 \leq \lambda<2 \pi$. This function is referred to as the transfer function of the system. Cosinusoids, $\exp \{i \lambda t\}$, are seen to be carried into cosinusoids. A variety of physical systems have this property to a good approximation.

Nonlinear time invariant systems may sometimes be approximated by Volterra expansions of the form

$$
Y_{t}=\sum_{s=-\infty}^{\infty} c_{t-s} X_{s}+\sum_{s=-\infty}^{\infty} \sum_{s^{\prime}=-\infty}^{\infty} d_{t-s, t-s^{\prime}} X_{s} X_{s^{\prime}}+\cdots
$$

The input $X_{t}=\exp \{i \lambda t\}$ here leads to the output

$$
C(\lambda) e^{i \lambda t}+D(\lambda, \lambda) e^{i 2 \lambda t}+\cdots
$$

where $C(\lambda)$ is given above and

$$
D(\lambda, \mu)=\sum_{s} \sum_{s^{\prime}} e^{-i \lambda s-i \mu s^{\prime}} d_{s, s^{\prime}}
$$

In such a nonlinear system one sees harmonics of the frequencies in the input appearing in the output. The laser example of Sec. 2 involved a system that was quadratic. 
Fourier analysis is useful in work with constant coefficient differential equations. These show the occurrence of oscillations and are often effective models of physical systems. Consider for example the linear system

$$
\frac{d \mathbf{S}(t)}{d t}=\mathbf{A} \mathbf{S}(t)+\mathbf{B} X(t)
$$

with $\mathbf{S}(\cdot)$ vector-valued and $X(\cdot)$ scalar. Supposing

$$
\mathbf{S}(t)=\int e^{i t \lambda} \mathbf{s}(\lambda) d \lambda
$$

and

$$
X(t)=\int e^{i t \lambda} x(\lambda) d \lambda
$$

by Fourier analysis one has the solution directly as

$$
\mathbf{S}(\lambda)=(i \lambda \mathbf{I}-\mathbf{A})^{-1} \mathbf{B} x(\lambda)
$$

Supposing $x(\lambda)$ constant and the latent values, $\mu_{j}$, of $\mathbf{A}$ to be distinct this may be written

$$
\mathbf{S}(t)=\sum_{j} \mathbf{a}_{j} e^{i \mu_{i} l}
$$

for some vectors $\mathbf{a}_{j}$. One sees the occurrence of oscillations at frequencies $\operatorname{Re} \mu_{j}$. One reference concerning such differential equations is Hochstadt (1964).

Turning to a further technique of Fourier analysis, that will be basic in one of the examples below, suppose that one is considering, for large $x$, an integral of the form

$$
\int e^{i k(\lambda) x} R(\lambda) d \lambda
$$

The method of stationary phase approximates this by

$$
e^{\operatorname{sgn} k^{\prime \prime}\left(\lambda_{0}\right) i \pi / 4} \sqrt{2 \pi /\left(x \mid k^{\prime \prime}\left(\lambda^{0} \mid\right)\right.} R\left(\lambda_{0}\right) e^{i k\left(\lambda_{0}\right) x} .
$$

where $\lambda_{0}$ satisfies $k^{\prime}\left(\lambda_{0}\right)=0$. References include Barndorff-Nielsen and Cox (1989) and Aki and Richards (1980). The idea is that unless the $k(\lambda)$ is near 0 the rapidly oscillating multipliers $\cos k(\lambda), \sin k(\lambda)$ will give the integral value 0 . 


\subsection{The Wavelet Case}

Wavelet analysis is enjoying a surge of contemporary investigation and is a competitor of Fourier analysis. It may be viewed as Fourier analysis with the sine and cosine functions replaced by other families of (orthogonal) functions. There are many similarities between Fourier and wavelet analysis. Consider the expansion in Eq. (3.2) with the coefficients in Eq. (3.1). The expansion is based on the fact that the sine and cosine functions provide a basis for $L_{2}[0,2 \pi)$. In wavelet analysis other systems of functions are used, see e.g., Strichartz (1993), Benedetto and Frazier (1994). Wavelets are of practical importance because they can sometimes provide more parsimonious descriptions than Fourier ones.

Wavelets often focus on local versus global behavior and in particular can pick up transient behavior. Basic is a (mother) wavelet $\psi(\cdot)$ nonzero only on say the unit interval $[0,1)$. Given a square-integrable function $g(x)$, one considers an expansion

$$
g(x)=\sum_{j=-\infty}^{\infty} \sum_{k=-\infty}^{\infty} \beta_{j k} \psi_{j k}(x)
$$

with

$$
\psi_{j k}(x)=2^{j / 2} \psi\left(2^{j} x-k\right)
$$

and

$$
\beta_{j k}=\int \psi_{j k}(x) g(x) d x
$$

The family $\left\{\psi_{j k}(\cdot)\right\}$ is taken to be orthonormal and complete, see e.g., Daubechies (1992), Walter (1992, 1994), Strichartz (1993), Benedetto and Frazier (1994).

The expansion in Eq. (3.7) represents $g(\cdot)$ in terms of functions with support individually on dyadic intervals $\left[k / 2^{j},(k+1) / 2^{j}\right]$ for $j, k$ integers. It suggests an approximation

$$
g^{J K}(x)=\sum_{\forall \mid \leq J} \sum_{|k| \leq K} \beta_{j k} \psi_{j k}(x)
$$

to $g(x)$. This may be written as

$$
g^{J K}(x)=\int W^{J K}(x, y) g(y) d y
$$

the kernel being

$$
W^{J K}(x, y)=\sum_{j, k} \psi_{j k}(x) \psi_{j k}(y)
$$


This kernel will tend to a delta function in various circumstances, see Walter (1992). Equation (3.10) can be used to study the degree of approximation directly as could Eq. (3.5) in the Fourier case. Equations (3.10) and (3.11) are wavelet analogs of Eqns. (3.4) and (3.5).

In the case of a discontinuous function, as will occur in Example 5.4, a particular wavelet analysis is especially suitable, namely Haar wavelet analysis. This analysis is based on the function

$$
\begin{aligned}
\psi(x)=1 & \text { for } 0 \leq x<\frac{1}{2} \\
-1 & \text { for } \frac{1}{2} \leq x<1 \\
0 & \text { otherwise }
\end{aligned}
$$

In the Haar case the kernel is

$$
W_{n}(x, y)=2^{n} \phi\left(2^{n} y-\left[2^{n} x\right]\right)
$$

with [.] here referring to integral part and $g_{n}(x)$ of Eq. (3.10) a local mean, $g_{n}(x)=|I|^{-1} \int_{I} g(y) d y, \quad x$ being in the particular interval $I=\left[m / 2^{\prime \prime},(m+1) / 2^{n}\right)$, see Fine (1949), Walter (1992).

There are empirical versions of Eq. (3.8) for use when discrete time data $Y_{t}, t=0, \ldots, T-1$ are available. One computes for example

$$
\hat{\beta}_{j k}=\frac{1}{T} \sum_{t=0}^{T-1} \psi_{j k}(t / T) Y_{t}
$$

Just as there are fast Fourier transforms, there are fast wavelet transforms, Strang (1993). Also one can write $p 2^{j}$ for $2^{j}$ above, with no real change in concept, but improved approximations in practice. The dynamic spectrum analysis of Example 5.2 is one type of wavelet analysis with $j=j_{0}$ and $\psi(x)=\exp \{-i 2 \pi x\}$.

Insertion of multipliers, as in Eq. (3.4) for Fourier approximation, is fundamental. This will be discussed later.

\section{STOCHASTICS AND STATISTICS}

In this section the quantities being transformed will be random.

\subsection{Stationary Processes}

Fourier analysis is basic to dealing with stationary random processes. A process, $Y_{t}$, is said to be second-order stationary if $\operatorname{cov}\left\{Y_{t+u}, Y_{t}\right\}$ exists for $t, u=0, \pm 1, \pm 2, \ldots$ and does not depend on $t$. In practice this often appears a reasonable working assumption. In the case of 
$Y_{t}, t=0, \pm 1, \pm 2, \ldots$ a second-order stationary process, following Cramér (1942), one has the Fourier representation

$$
Y_{t}=\int_{-\pi}^{\pi} e^{i t \lambda} d Z(\lambda)
$$

with $Z(\cdot)$ a random function such that

$$
\operatorname{cov}\{d Z(\lambda), d Z(\mu)\}=\delta(\lambda-\mu) f(\lambda) d \lambda d \mu
$$

$-\pi<\lambda, \mu \leq \pi, f(\cdot)$ being the power spectrum of $Y$ and $\delta(\cdot)$ the Dirac delta function. The Cramér representation has the advantage of taking one directly to the Fourier domain and thereby making some operations on the process clearer. The series $Y_{t}$ may be vector-valued. Then the crossspectral density matrix, $\mathbf{f}(\cdot)$, is given by

$$
\operatorname{cov}\{d \mathbf{Z}(\lambda), d \mathbf{Z}(\mu)\}=\delta(\lambda-\mu) \mathbf{f}(\lambda) d \lambda d \mu
$$

Cross-spectrum analysis is useful for system analysis, i.e., estimating for example the transfer function of a linear time invariant system.

Higher-order spectra may be defined directly via $Z(\cdot)$, e.g., the bispectrum $f(\lambda, \mu)$ at frequency $\lambda, \mu$ is given by

$$
\operatorname{cum}\{d Z(\lambda), d Z(\mu), d Z(\nu)\}=\eta(\lambda+\mu+\nu) f(\lambda, \mu) d \lambda d \mu d \nu
$$

where $\eta(\lambda)$ is the $2 \pi$ periodic extension of the Dirac delta function.

Empirical Fourier analysis, e.g., of residuals of a fit, provides a diagnostic using in particular the result that if the process is white noise, the power spectrum is constant in frequency, $\lambda$.

Blackman and Tukey (1959), Båth (1974), Brillinger (1975) and Bloomfield (1976) are books focussing on the empirical Fourier analysis of time series

\subsection{Central Limit Theorems}

In classic forms the central limit theorem is concerned with the distributions of sums of independent random variables

$$
S_{T}=Y_{0}+Y_{1}+\cdots+Y_{T-1}
$$

and their approximate normality with variance $T \sigma^{2}$ for large $T$. It is usual to assume that the $Y$ 's are identically distributed.

At some point engineers began promulgating a folk theorem to the effect that narrow-band noise is approximately Gaussian, [see Leonov and Shiryaev (1960), Picinbono (1960), Rosenblatt (1961)]. One fashion to formulate this remark is as a statement that

$$
S_{T}(\lambda)=Y_{0}+e^{-i \lambda} Y_{1}+\cdots+e^{-i \lambda(T-1)} Y_{T-1}
$$


$0 \leq \lambda<2 \pi$, is approximately (complex) normal for each $\lambda$. Under stationarity and mixing assumptions for the series $Y_{t}$, the variance of Eq. (4.2) is approximately

$$
2 \pi T f(\lambda)
$$

with $f(\lambda)$ the power spectrum of Eq. (4.1) at frequency $\lambda$. Surprisingly, the values of $S_{T}(\lambda)$ at distinct frequencies of the form $\lambda=2 \pi j / T$, are approximately independent. Problems involving stationary mixing processes may thus be converted into ones involving (approximately) independent normal random variables. Empirical Fourier transforms such as Eq. (4.2) have many uses and several are indicated in this paper. A fundamental use is to estimate a power spectrum by smoothing the squared-modulus.

Early work on the asymptotic properties of finite Fourier transforms includes that of Slutsky (1934), Leonov and Shiryaev (1960), Rosenblatt (1961), Good (1963), Hannan (1969), Brillinger (1969), Hannan and Thomson (1971), Hannan (1972).

There has been some consideration of the cases of long range dependence and stable distributions. References include: Rosenblatt (1981), Freedman and Lane (1981), Fox and Taqqu (1986), Yajima (1989), Shao and Nikias (1993). The case of random generalized functions, which includes for example point processes and random measures, is considered in Brillinger (1982).

In the case of wavelets and a model

$$
Y_{t}=g(t / T)+\varepsilon_{t}
$$

with $\varepsilon_{t}$ stationary noise having power spectrum $f(\lambda)$, under regularity conditions, the statistic $\hat{\beta}_{j k}$ of Eq. (3.13) may be shown to be asymptotically normal with mean $\beta_{j k}$ and variance

$$
\frac{2 \pi}{T} f(0)
$$

see Brillinger (1996). The variance is the same as that of Eq. (4.4). Further when the functions $\psi_{j k}(\cdot)$ and $\psi_{j^{\prime} k^{\prime}}(\cdot)$ are orthogonal, the coefficients $\hat{\beta}_{j k}, \hat{\beta}_{j^{\prime} k^{\prime}}$ are approximately independent for distinct $(j, k)$ and $\left(j^{\prime}, k^{\prime}\right)$. This last results suggests that an estimate of $f(0)$ may be obtained by averaging the values $T\left|\hat{\beta}_{j k}\right|^{2} / T$ for which $\beta_{j k}=0$.

\subsection{Shrinking}

Among surprises in working with Fourier transforms is the importance of convergence factors. These are the $w_{k}^{K}$ of Eq. (3.4). In Eq. (3.4) they shrink the coefficients of the $\exp \{i x k\}$ towards 0 as $k$ increases. Such multipliers are 
also important in the stochastic case, see: Tukey (1959), Brillinger (1975), Bloomfield (1976), Dahlhaus (1984, 1989).

A related concept is shrinking. In a regression context Tukey (1979) distinguishes three types of shrinking. Crudely: "first shrinkage" corresponds to pretesting and selection of regressor variables, "second shrinkage" corresponds to a type of Wiener filtering and "third shrinkage" corresponds to borrowing strength from other coefficients to improve the collection of coefficients. In this last case the multipliers are not meant for attenuating high frequencies, rather they are meant for attenuating uncertain terms. A common characteristic is that the estimates become biased; however, biased estimates have long been dominant in time series analysis.

Second shrinkage plays an important role in two of the examples that follow. A particular second shrinkage estimate, introduced in Tukey (1979), may be motivated as follows. Consider a classic simple regression model

$$
y=\beta x+\varepsilon
$$

with $b$ an estimate of $\beta$ and $s$ an estimate of its standard error. Seek a multiplier $m$ such that $m b x$ is an improved estimate of $\beta x$. The meansquared error of the new estimate is

$$
x^{2} E\left\{(\beta-m b)^{2}\right\}
$$

which may be estimated by

$$
x^{2}\left\{(1-m)^{2}\left[b^{2}-s^{2}\right]+m^{2} s^{2}\right\}
$$

This is minimized by the choice $m=1-s^{2} / b^{2}$. One would prefer to take $m$ to be the positive part

$$
\left(1-s^{2} / b^{2}\right)_{+}
$$

This multiplier has the reasonable property of being 0 for $b$ less than its standard error.

In Sec. 3.1 convergence factors, $w_{k}^{K}$, were inserted into trigonometric expressions to obtain improved approximation. In Example 5.1 such multipliers based on the reliability of estimated coefficients $\hat{c}_{k}$ will be inserted to obtain an improved estimate. To estimate $\hat{g}(x)$ of Eq. (4.4) one considers, for example,

$$
\hat{g}(x)=\sum_{k} w\left(\hat{c}_{k} / s_{k}\right) \hat{c}_{k} e^{i x k}
$$

where $s_{k}^{2}$ is an estimate of the variance of $\hat{c}_{k}$ and $w(u)$ is a function that is near 1 for large $u$ and near 0 for small $u$. Examples of functions $w(\cdot)$ are given in Fig. 1. 


\section{Shrinkage factor}

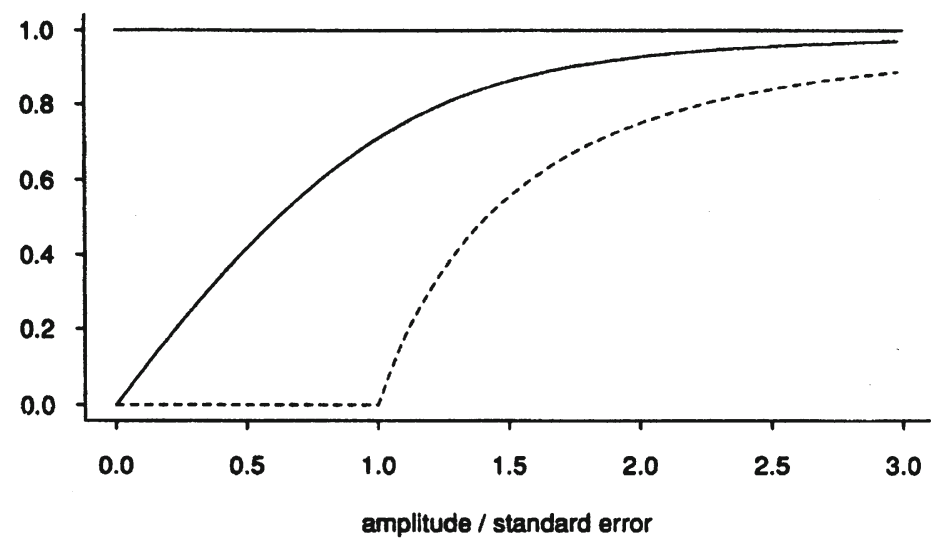

Figure 1. Graph of the multipliers Eqns (5.7) and Eq. (5.8), as a functions of the amplitude of the estimate divided by its estimated standard error.

In work to obtain improved wavelet-based estimates, Donoho and Johnstone (1990), Hall and Patil (1995) create shrinkage estimates involving multipliers, there referred to as "thresholders". The estimates take the form

$$
\sum_{|j| \leq J} \sum_{|k| \leq K} w\left(\left|\hat{\beta}_{j k}\right| / s_{j k}\right) \hat{\beta}_{j k} \psi_{j k}(x)
$$

where $s_{j k}$ is an estimate of $\operatorname{var} \hat{\beta}_{j k}$ and $0 \leq w(\cdot) \leq 1$.

There are many classical references to selection of variables and pretesting, i.e., first shrinkage. References to second shrinkage include: Whittle (1962), Thompson (1968), King (1972), Ott and Kronmall (1976), Tukey (1979), Zidek (1983), Donoho and Johnstone (1990), Stoffer (1991), Hall and Patil (1993), Donoho et al. (1995). References to third shrinkage include: Stein (1955), Efron and Morris (1977), Copas (1983), Saleh (1992).

\section{EXAMPLES}

In this Section four biological and physical examples are presented.

\subsection{Electron Microscopy}

Electron microscopy is tool for studying the placements of atoms within molecules. It is mainly carried out with crystalline (periodic) material. One 
problem is to obtain improved images and that is the concern of the present example. Glaeser (1985), Henderson et al. (1986), Hovmöller (1990) are references describing the basics of electron microscopy.

In the planar case, the principal theoretical concept is the projected (Coulomb) density distribution

$$
V(x, y)=\sum_{h, k} F_{h, k} e^{2 \pi i(h x+k y) / \Delta}
$$

$h, k=0, \pm 1, \pm 2, \ldots$ with $(x, y)$ planar coordinates and with $\Delta$ the period of the crystal. The function $V(\cdot)$ is real-valued and has various symmetries. The $h, k$ in Eq. (5.1) are referred to as the Miller indices, while the $F_{h, k}$ are referred to as structure factors. One wishes to estimate $V(x, y)$ over $0 \leq x, y<\Delta$.

The datum is an image, $Y(x, y)$, with $0 \leq x<X, 0 \leq y<Y$. The image may be written as

$$
Y(x, y)=V(x, y)+\text { noise }
$$

The empirical Fourier transform is

$$
\hat{F}_{h, k}=\int_{0}^{Y} \int_{0}^{X} Y(x, y) e^{-2 \pi i(h . x+k y) / \Delta} d x d y
$$

which may be written

$$
\int_{0}^{\Delta} \int_{0}^{\Delta} \sum_{m, n} Y(x+m \Delta, y+n \Delta) e^{-2 \pi i(h x+k y) / \Delta} d x d y
$$

The synthesis corresponding to the analysis Eq. (5.3) is

$$
\sum_{h, k} \hat{F}_{h, k} e^{2 \pi i(h x+k y) / \Delta}
$$

$0 \leq x<\Delta, 0 \leq y<\Delta$.

There has been concern to form an improved image. In this connection Blow and Crick (1959), Hayward and Stroud (1981) introduced "multipliers", $w(\cdot)$, into expressions like Eq. (5.5), forming

$$
\hat{V}(x, y)=\sum_{h, k} w\left(\left|\hat{F}_{h, k}\right| \hat{\sigma}_{h, k}\right) \hat{F}_{h, k} e^{2 \pi i(h x+k y) / \Delta}
$$

where the $\hat{\sigma}_{h, k}$ are estimates of the standard errors of the $\hat{F}_{h, k}$. This is a second shrinkage estimate. Consideration of the mean-squared error, as in Eq. (4.5), leads to the multiplier

$$
w(|\hat{F}| / \hat{\sigma})=\left(1-\frac{\hat{\sigma}^{2}}{|\hat{F}|^{2}}\right)_{+}
$$


which by analogy with Wiener filtering will be called the Wiener multiplier. By Bayesian arguments Blow and Crick (1959) and Hayward and Stroud (1981) were lead to the multiplier

$$
w(\gamma)=\frac{\sqrt{\pi}}{2} \gamma\left[I_{0}\left(\frac{\gamma^{2}}{2}\right)+I_{1}\left(\frac{\gamma^{2}}{2}\right)\right] e^{-\gamma^{2} / 2}
$$

with $\gamma=|\hat{F}| / \hat{\sigma}$, and $I_{0}, I_{1}$ modified Bessel functions, see Brillinger et al. (1989, 1990). It and Eq. (5.7) are graphed in Fig. 1. These multipliers approach 1 as the uncertainty approaches 0 .

Estimates employing Eqns (5.7) and (5.8) are illustrated in Fig. 2 for images of the protein bacteriorhodopsin. This substance occurs naturally

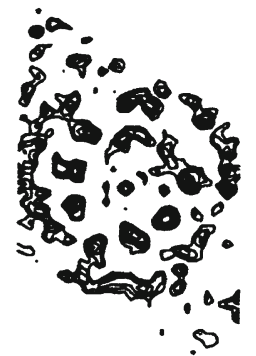

Naive image

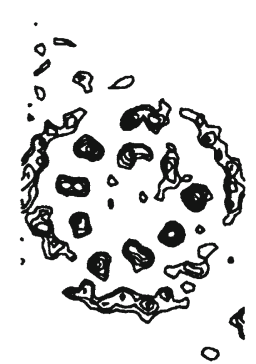

Blow-Crick image

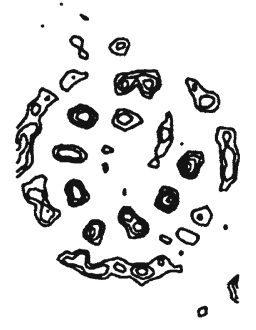

Wiener image

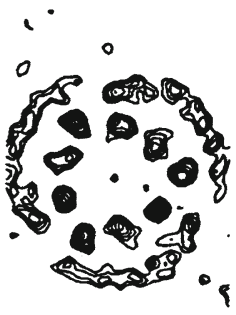

Final image, $n=42$

Figure 2. Estimates of the basic cell of bacteriorhodopsin. The upper left panel is the naive estimate as shown in Eq. (5.5). The upper right panel is the estimate Eq. (5.6) with the multiplier, Eq. (5.8). The bottom left panel is the estimate Eq. (5.6) with the multiplier, Eq. (5.7). The last panel is Eq. (5.6), with Eq. (5.8), obtained by combining 42 individual images. 
as a two-dimensional crystalline array within the cell membrane of Halobacterium halobrium. Together with accompanying lipid molecules, it is known as "purple membrane". This crystal is based on a hexagonal lattice. In Fig. 2 only the positive contours are shown. (Negative density features signify the absence of atoms and thus have no direct usefulness when the density map is interpreted.) The first panel of Fig. 2 shows the elementary estimate of Eq. (5.5). The top right shows Eq. (5.6) with $w(\cdot)$ of Eq. (5.7). The third, lower left, shows Eq. (5.6) with $w(\cdot)$ of (5.8). The final panel provides an estimate based on combining 42 individual images. This last image may be viewed as what the earlier estimates based on a single image ascribe to be.

Through the inclusion of the multipliers, the peaks have become more substantial and better separated. Also, the estimates show better approximations to a three-fold symmetry. Details of the data collection and further details of the analysis may be found in Brillinger et al.. (1989, 1990).

The Fourier transform is useful in this example firstly because of the lattice periodicities and secondly for the central limit theorem result suggesting specific estimates of the $s_{h k}$ of Eq. (5.6) namely for $s_{h k}^{2}$ one takes the average of the squared moduli of Fourier coefficients thought to be signal free.

There are extensions to the 3D case, see Henderson et al. (1990), Wenk et al. (1992).

\subsection{Seismic Surface Waves}

Various sound waves are transmitted through the Earth following a seismic disturbance, in particular surface (or Rayleigh) waves. These are vibrations whose energy is trapped and propagated just under the surface. The waves have sinusoidal form and are prominent in the later part of a seismogram. For example see Fig. 3 for an event that was recorded in Uppsala, Sweden. These waves have the interesting aspect of having been discovered mathematically. For basic details see Aki and Richards (1980) and Bullen and Bolt (1985).

Consider modelling that part of a seismogram where the Rayleigh waves occur. Let $Y(x, t)$ denote the vibrations recorded at distance $x$ from the earthquake source, as a function of time $t$. With a layered crust model the theoretical seismogram is a solution of a system of differential equations with associated boundary conditions and may be represented as

$$
\int e^{-i(\lambda t-k(\lambda) \cdot x)} R(\lambda) d \lambda
$$




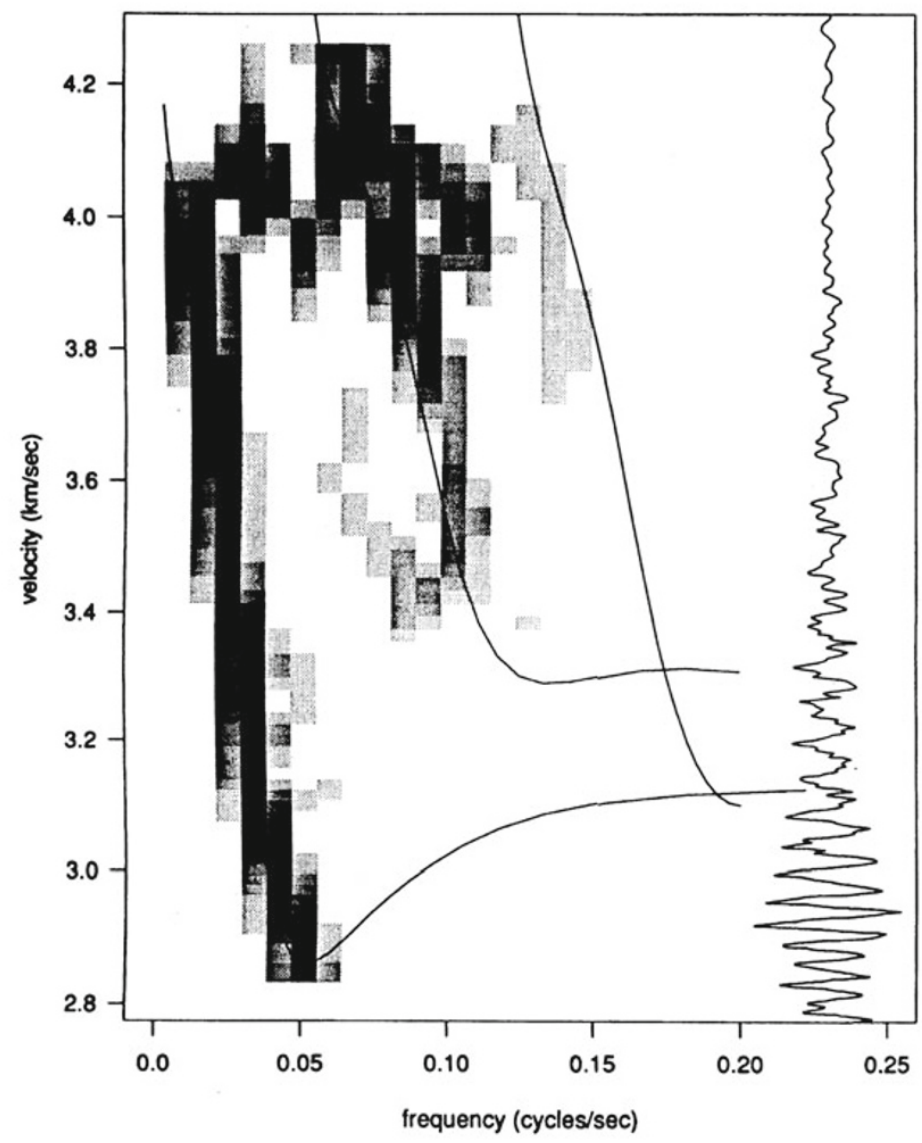

Figure 3. The Siberia-Upsalla dynamic spectrum as a function of frequency and velocity as computed from Eq. (5.11). The vertical trace is the seismogram as a function of velocity.

Here, when $x=0$

$$
\int e^{-i \lambda t} R(\lambda) d \lambda
$$

represents the vibrations at the earthquake source. The solution in Eq. (5.9) comes from substituting a particular solution $\exp \{-i(\lambda t-k x)\}$ into the differential equations and matching boundary conditions, see Aki and Richards (1980). One writes $k(\lambda)=\lambda / c(\lambda)$ with $c(\lambda)$ the (phase) velocity 
with which the wave of frequency $\lambda$ travels. The functions $k(\cdot)$ and $c(\cdot)$ depend on the transmission medium.

In the case that $x$ is large one can use the method-of-stationary phase, described in Section 3.1, to see the sinusoidal form of the waves. Specifically for large $x$, Eq. (5.9) is approximately

$$
R\left(\lambda_{t}\right) \exp \left\{-i\left(\lambda_{t} t-k\left(\lambda_{t}\right) x\right)\right\}
$$

with $\lambda_{t}$ the solution of

$$
\frac{d}{d \lambda}\{\lambda t-k(\lambda) x\}=0
$$

that is $k^{\prime}\left(\lambda_{t}\right)=t / x=1 / U\left(\lambda_{t}\right)$. Here $U(\lambda)$ is the group velocity, the velocity with which the energy travels, at frequency $\lambda$. The phenomenon of waves with different frequencies travelling with different velocities, as occurs here, is called dispersion.

Given an earth model, $\theta$, that is a collection of layer depth, velocity and density parameters, one can compute the group velocity $U(\lambda \mid \theta)$, see Bolt and Butcher (1960), Aki and Richards (1980). For frequency $\lambda$ and parameter $\theta$ there may be several possible dispersion curves $U_{n}(\lambda \mid \theta), n=0,1,2, \ldots$. called modes. Dynamic Fourier analysis provides a way to see these modes, and is presented in Fig. 3. The concern of the example of this section is to estimate $\theta$.

The event studied originated in Siberia, 20 April 1989, and the trace was recorded at Uppsala, Sweden. Figure 3 provides a grey scale display of energy as a function of velocity and frequency. It is computed as

$$
\left|\sum_{s=-S}^{S} h(s / S) Y(t-s) e^{-i s \lambda}\right|^{2}
$$

with $t=x_{0} / v, v$ velocity, $x_{0}$ distance to source and $h(\cdot)$ a convergence factor. One sees waves of about 0.07 cycles/second arriving first. Figure 3 also shows the dispersion curves $U_{n}(\lambda \mid \hat{\theta})$ for one fitted earth model. Some further details are given in Brillinger (1993).

The velocity-frequency curves, $U_{n}(\lambda \mid \theta)$, may be inverted to frequencytime curves $\lambda=\lambda_{l}(t \mid \theta)$. To estimate $\theta$ one can then consider choosing $\theta, \alpha$ to minimize

$$
\sum_{t}\left|Y(t)-\int e^{-i\left(\lambda t-k(\lambda \mid \theta) x_{0}\right)} R(\lambda \mid \alpha) d \lambda\right|^{2}
$$

where $\alpha$ is some parametrization of the source motion. One approach is to approximating the integral in Eq. (5.9), is to take $R(\cdot)$ piecewise constant, linear in $\alpha$. Figure 4 provides the results of such an analysis. Graphed are 
Siberian Event at Uppsala April 20,1989

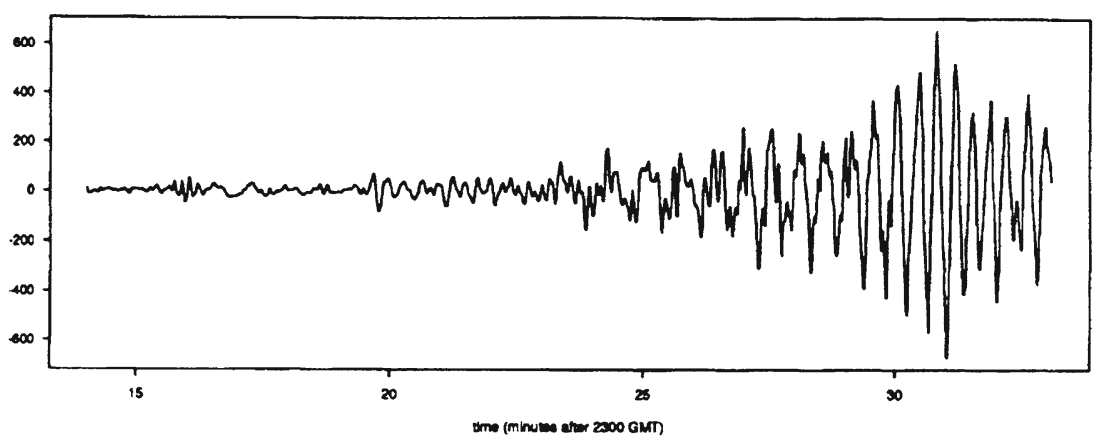

Fit

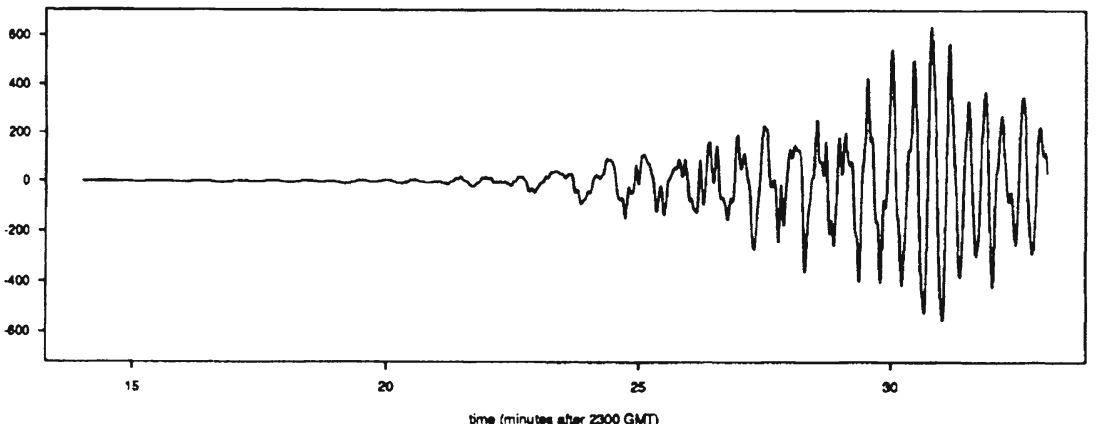

Residuals

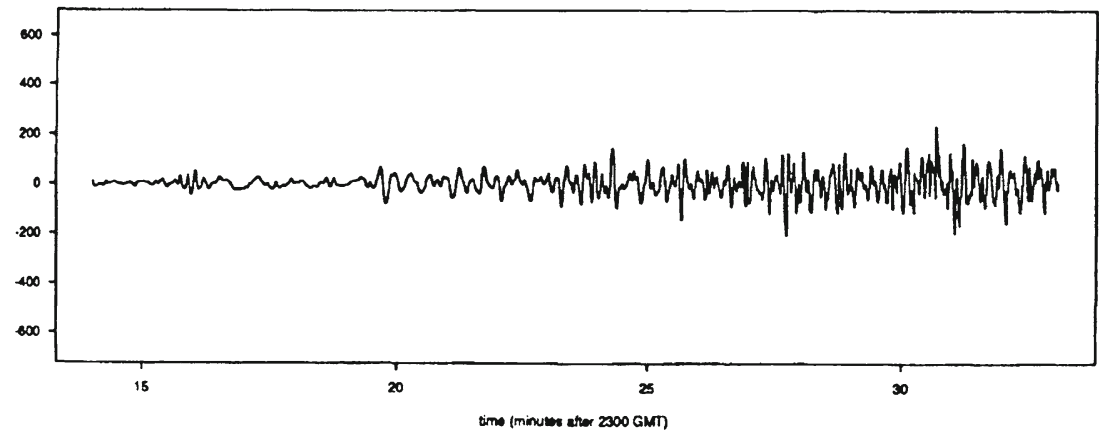

Figure 4. The top trace is the seismogram as a function of time. The middle is the fit based on Eq. (5.9). The bottom is the difference of these two. 
the series, the fit and the residuals. The standard errors might be computed as in Richards (1961), focussing on the nonlinear parameters $\theta$ and acting as if the noise series was white. An improved estimation procedure is needed, for the residual series of Fig. 4 suggests the presence of signal-generated noise.

Even though this particular situation is clearly nonstationary, Fourier analysis has been basic to addressing it. This is a consequence of the presence of dispersion. The example is also of additional interest since one has a Fourier transform of two variables whose support lies on several curves, see Fig. 3. This type of plot allows inference of the presence of higher modes and assessment of the fit as well.

\subsection{NMR Spectroscopy}

Nuclear magnetic resonance is a quantum mechanical phenomenon employed to study the structure of various molecules. In an experiment, one creates a fluctuating magnetic field, $X(t)$, encompasing a substance and then observes an induced voltage, $Y(t)$. Hennel and Klinowski (1993) is one general reference.

If $\mathbf{S}(t)$ is a vector describing the state of the system at time $t$, then the fluctuations are described by the Bloch equations

$$
\frac{d \mathbf{S}(t)}{d t}=\mathbf{a}+\mathbf{A S}(t)+\mathbf{B S}(t) X(t)
$$

and the measurements by

$$
Y(t)=\mathbf{c}^{\tau} \mathbf{S}(t)+\text { noise }
$$

with $\mathbf{c}$ depending on the geometry of the experiment. The principal parameters are frequencies of oscillation and decay rates. The parameters of interest sit in the matrices A and B, see Brillinger and Kaiser (1992). The entries of $\mathbf{A}$ and $\mathbf{B}$ have physical interpretations, e.g., the diagonal entries of A represent occupancy probabilities.

Equations (5.12) is interesting for being bilinear. It can be solved, symbolically, by successive substitutions, obtaining

$$
\mathbf{S}(t)=\mathbf{C}+\int^{t} e^{\mathbf{A}(t-s)} \mathbf{C} X(s) d s+\int^{t} \int^{s} e^{\mathbf{A}(t-s)} \mathbf{B} e^{A(s-r)} \mathbf{C} X(r) X(s) d r d s+\cdots
$$

with $\mathbf{C}=-\mathbf{A}^{-1} \mathbf{a}$. If $\mathbf{A}$ is written $\mathbf{U} e^{\Lambda} \mathbf{U}^{-1}$ with $\Lambda$ diagonal, then the pulse response, $\mathbf{S}(t)$, is seen to be a sum of complex exponentials and various of their powers and products. The real parts of the entries of $\Lambda$ will lead to the decay of these components while the imaginary parts represent resonance frequencies. 
The problem is to estimate the parameters of Eq. (5.12) and thereby, to characterize the substance. Some of the parameters may be estimated by cross-spectral analysis and others by likelihood analysis.

Brillinger and Kaiser (1992) present results from a study of 2,3-dibromothiophene. The matrices $\mathbf{A}$ and $\mathbf{B}$ are $4 \times 4$ with complex-valued entries. The parameters include a coupling constant, $J$ and frequencies $\omega_{A}$ and $\omega_{B}$. In the experiment the input employed was a sequence of pulses

$$
X(t)=\sum_{j} M_{j} \delta(t-j \Delta)
$$

with $\Delta=1 / 150 \mathrm{~s}, t$ in seconds and $M_{j}$ the $m$-sequence given by $M_{j}=M_{j-1} M_{j-4} M_{j-8} M_{j-12}$ starting at $M_{j}=-1$ for $j=1, \ldots, 12$.

Figure 5 presents corresponding stretches of input and output together with the results of a cross-spectral analysis. Specifically the first-order transfer function estimate

$$
\hat{A}(\lambda)=\frac{\operatorname{smooth}_{\mu \approx \lambda}\left\{\left[\sum_{t} Y(t) e^{-i \mu t}\right] \overline{\left[\sum_{t} X(t) e^{-i \mu t}\right]}\right\}}{\operatorname{smooth}_{\mu \approx \lambda}\left\{\left|\sum_{t} X(t) e^{-i \mu t}\right|^{2}\right\}}=\hat{f}_{Y X}(\lambda) \hat{f}_{X X}(\lambda)^{-1}
$$

is given in Fig. 5. Theoretically its peaks are located at the frequencies

$$
\left(\omega_{A}+\omega_{B}\right) / 2 \pm J \pm \sqrt{J^{2}+\left(\omega_{A}-\omega_{B}\right)^{2} / 2}
$$

and the widths of the peaks relate to a damping constant $T_{2}$.

In a more detailed analysis the parameters of the model, including initial state values, were estimated by least squares seeking

$$
\min _{\theta} \sum_{t}\left|Y(t)-\mathbf{c}^{\tau} \mathbf{S}(t \mid \theta)\right|^{2}
$$

$\theta$ referring to the unknown parameters. In the computations the state vector, $\mathbf{S}(t \mid \theta)$ was evaluated recursively. Figure 6 shows the amplitude of the Fourier transform of the data and of the corresponding fit. (It is usual to graph an unsmoothed estimate in the NMR literature in order to obtain high resolution of peaks.) There is an intriguing small peak just above $60 \mathrm{~Hz}$ which recurs when the time series is broken down into contiguous segments. NMR researchers refer to such a phenomenon as a "birdie", but had no explanation for its source in the present case. Further details may be found in Brillinger and Kaiser (1992).

There are extensions of the cross-spectral approach to the $2,3,4, \ldots$ and higher dimensional cases, see Blümich (1985). 
Input

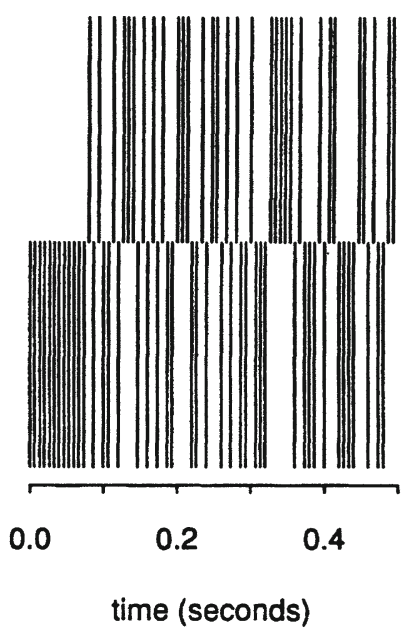

Output

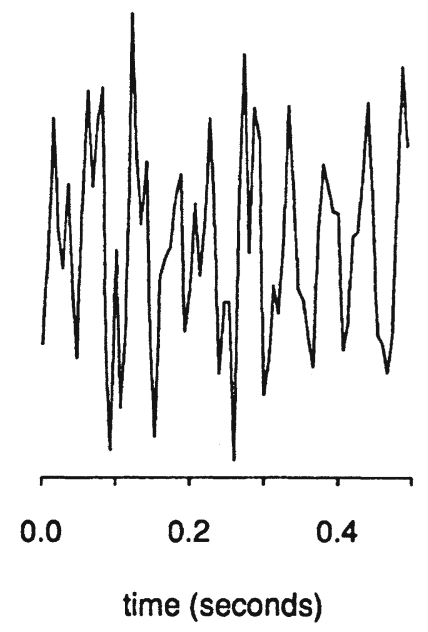

Modulus transfer function

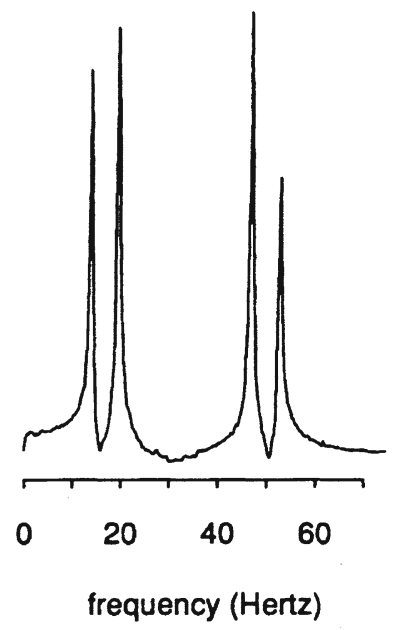

Phase transfer function

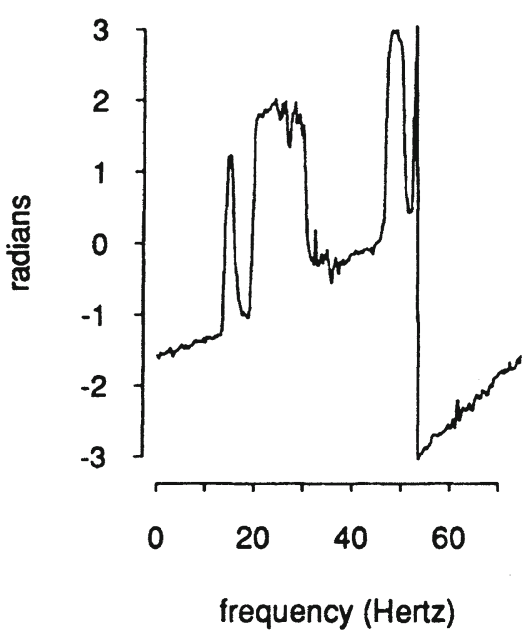

Figure 5. Results of a nuclear magnetic resonance study of 2,3-dibromothiophene. The top left is a segment of the input and below is the corresponding output. The right column provides the estimated amplitude and phase of the (linear) transfer function. 


\section{$\log 10|\mathrm{FT}|$ fit}
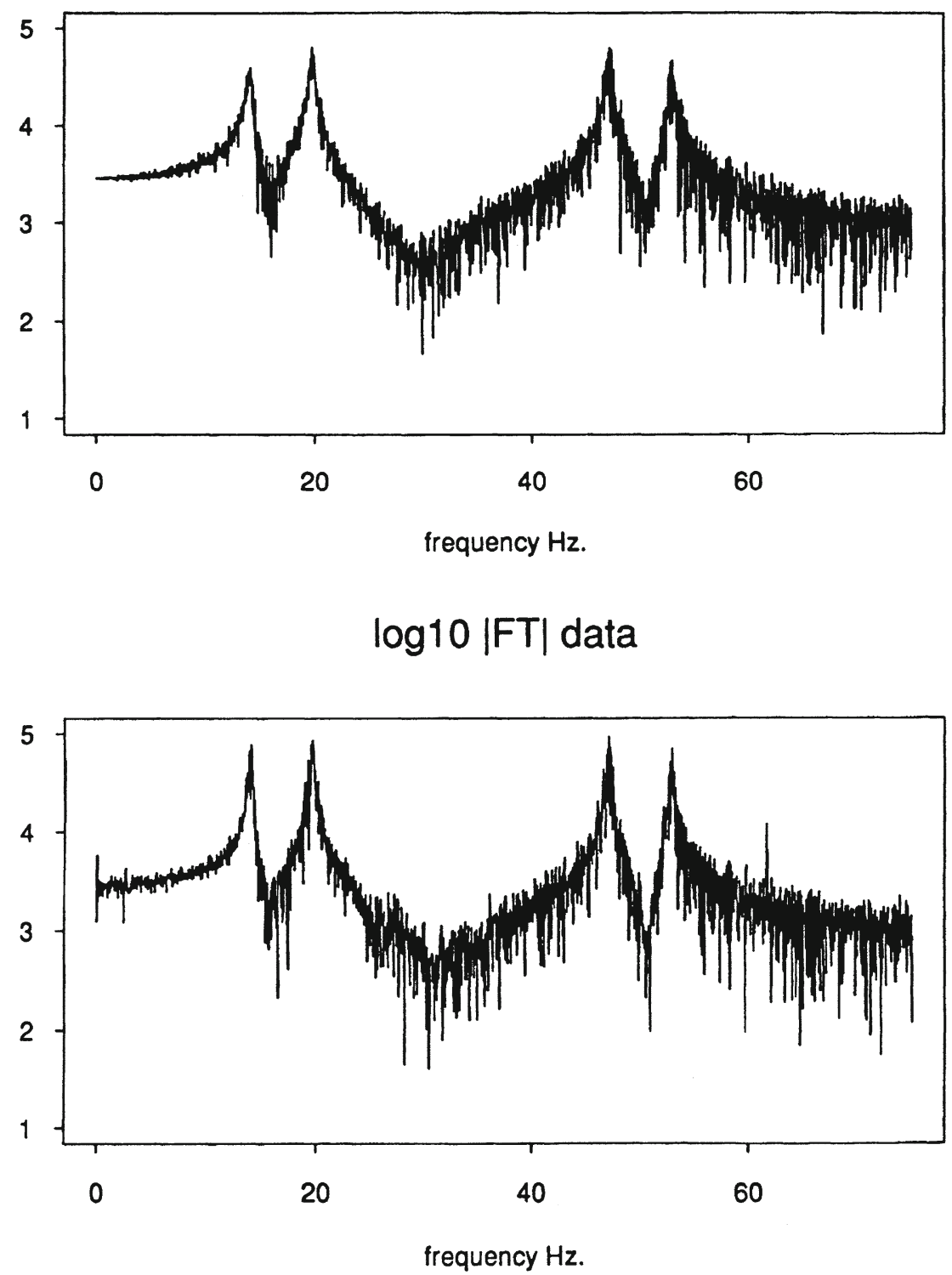

Figure 6. The modulus of the Fourier transform of the output and of the corresponding fit derived from Eq. (5.14). 

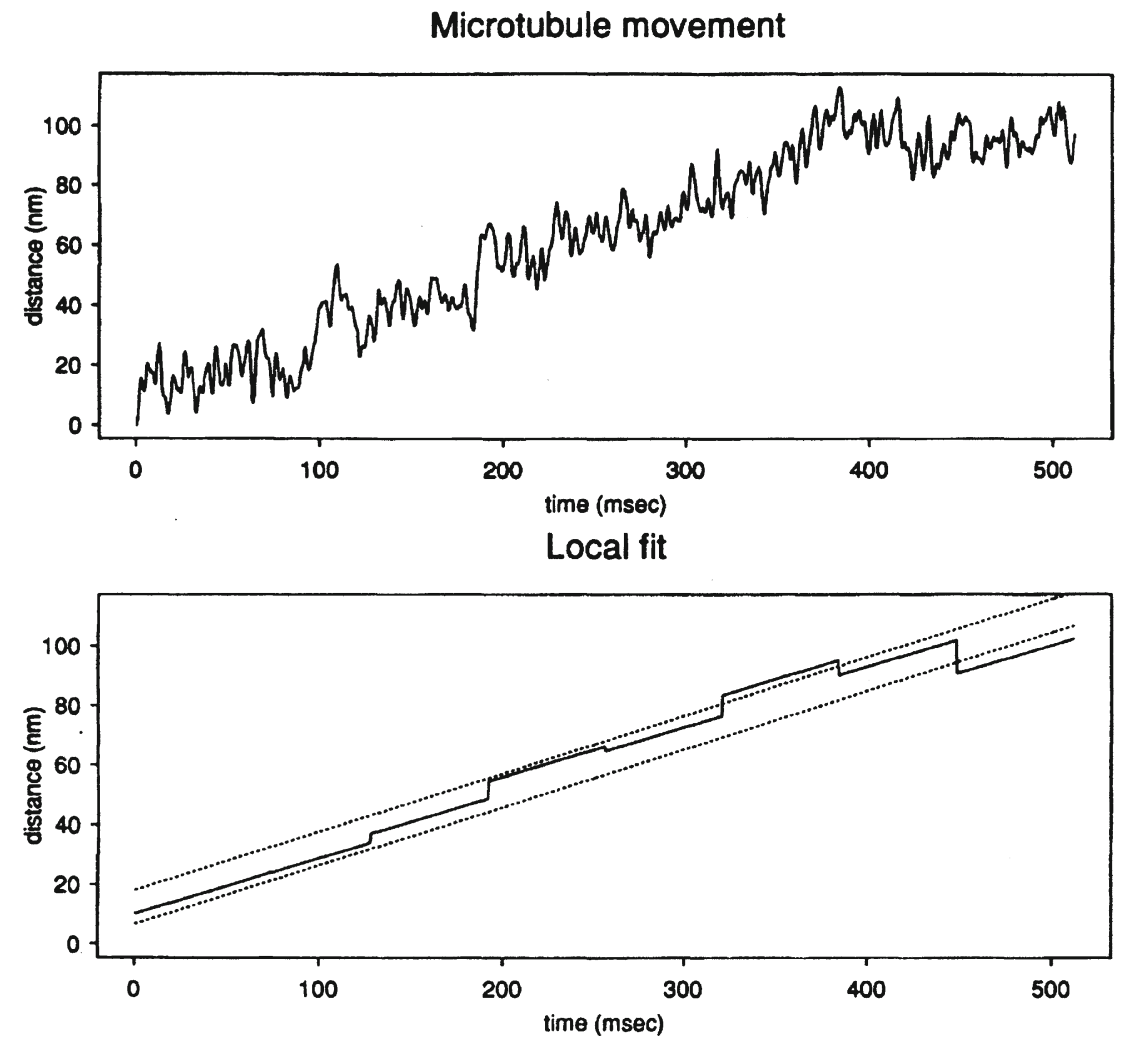

Improved local fit

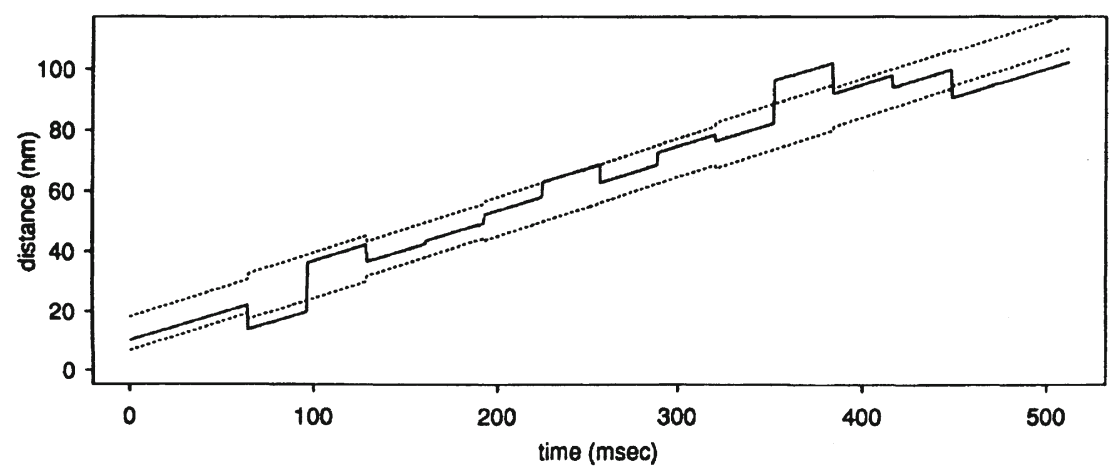

Figure 7. The top trace is the estimated movement of a microtubule as a function of time. The middle provides the fit with no shrinkage. The bottom panel provides a shrunken fit. The dashed lines provide approximate \pm 2 standard error limits. 
In this example the Fourier transform is useful for examining resonance, for assessing goodness of fit and for understanding the nonlinearity involved.

\subsection{Microtubule Movement}

As an illustration of wavelet analysis, consider the problem of searching for jumps in records of microtubule movement. Microtubules are linear polymers basic to cell motility. A concern is whether movement is smooth, or rather via a series of jumps, see Malik et al. (1994).

If $Y(t)$ denotes the distance a microtubule has travelled at time $t$, the model considered is

$$
Y(t)=\alpha t+g(t / T)+\text { noise }
$$

$t=0, \ldots, T-1$ with $\alpha$ a parameter related to diffusion motion and $g(\cdot)$ a step function. The model in Eq. (5.15) will be approximated by

$$
Y(t)=\alpha t+\sum_{k} \gamma_{n k} \phi_{n k}(t / T)+\text { noise }
$$

for some $n$. Because of the presence of the term $\alpha t$ in Eq. (5.16) the analysis in the present case is not so immediate, but still all that one needs are local means. The least squares estimates are obtained by regression of $Y$ on the $\phi_{n k}(t / T)$ and on $t$ made orthogonal to the $\phi_{n k}$. Further details on the fitting are given in the Appendix to this chapter.

In the experiments of concern samples were taken from the bovine brain. Specifics may be found in Malik et al. (1994). The top panel of Fig. 7 provides a data trace. Next is an estimate $\hat{g}_{n}(t / T)$ with $w(u)$, of Eq. (4.7), identically 1 . The final panel an improved estimate based on the multiplier $w(u)=\left(1-1 / u^{2}\right)_{+}$. The value of $n=3$ was chosen having in mind a search for isolated jumps for this particular data set. Also indicated are approximate \pm 2 standard error limits around the fitted straight line. There is little evidence for the presence of isolated jumps. The construction of the standard error estimate is described in the Appendix to this chapter.

The Fourier transform was used here to develop uncertainty estimates, following on an assumption that the noise was stationary.

\section{SOME OPEN PROBLEMS}

This Section briefly lists a number of topics, motivated by the examples of the paper, that appear fruitful for more development.

Foremost among the topics calling out for further research is the theoretical and practical development of shrinkage estimates. The ideas are basic. 
The effects are substantial, see Fig. 2 for example. One wonders about "optimal" choice of the multipliers/shrinkage factors. Perhaps optimal rates of convergence may be determined and then it be checked which multipliers lead to those. This paper has focused on second shrinkage. Berger and Wolpert (1983) develop third shrinkage estimates in random function cases. Lillestol (1977) studies time series in one case.

In both the surface wave and nuclear magnetic resonance examples, examination of residuals suggests the presence of signal-generated noise. Better estimates are needed. Either because the ones used are inefficient or because the signal-generated noise is basic. In the latter case an appropriate likelihood function needs to be developed. Ihaka (1993) does this for one case in seismology. If the noise is indeed nonstationary and autocorrelated, then a novel form of uncertainty estimation technique will be needed. In the case of the "improved" wavelet estimate, the uncertainty was estimated as if the shrinkage factors were constant, see Appendix to this chapter. Perhaps a useful bootstrap procedure could be developed, based on an assumption of stationary innovations being present.

Quite a different type of problem is the following: develop the aliasing structure for higher-order spectra in the case of a process observed on a lattice. This will be particularly complicated in the case of lattices in $R^{p}$ with $p>1$. Another problem in the case of image estimates, is how to visualize the associated uncertainty.

The Fourier transforms studied have all been scalar-valued. There are central limit theorems for processes taking on values in a group. It would be of interest to obtain corresponding results for group-valued Fourier transforms, e.g., in the p-adic case.

\section{DISCUSSION AND SUMMARY}

The principal interest of the examples of the paper has been in problem formulation and in addressing particular scientific questions. In each of the examples, an empirical Fourier transform has played a central role. With its broad collection of understood properties this transform has assisted the analyses greatly. The usefulness of second shrinkage, analogous to the use of convergence factors in Fourier approximation, is also noteworthy.

The particular groups of the examples have been abelian. General group theoretic ideas and empirical Fourier analysis have been discussed for other groups. For the case of the symmetric group see Diaconnis $(1988,1989)$ and Kim and Chapman (1993). For the locally compact abelian case see Brillinger (1982). For p-adics see Brillinger (1992). The use of $p$-adics in signal 
processing is discussed in Gorgui-Naguib (1990). For other cases see Hannan (1969). Key distinctions that arise are abelian versus nonabelian groups, compact versus locally compact groups, and whether $t$ is in a group or $Y$ is in a group.

There are other transforms that are useful in practice. These include: the Laplace, Hilbert, Stieltjes, Mellin, with some work having been done for abstract groups, see Loomis (1953).

The case of lacunary trigonometric series is somewhat like the case of point processes. Here the Fourier transform has a different form, e.g., for point process data $\left\{\tau_{1}<\tau_{2}<\cdots<\tau_{N}\right\}$ it is given by

$$
\sum_{j=1}^{N} \exp \left\{-i \lambda \tau_{j}\right\}
$$

$-\infty<\lambda<\infty$. Such a transform is used in Rosenberg et al. (1989) for example.

Unemphasized, but important, topics include: the Poisson summation formula useful in understanding aliasing and the sampling theorem (Hannan (1965)), abstract fast algorithms (Rockmore (1990)), spherical functions (Terras (1988)), uncertainty principles (Smith (1990)).

In conclusion we quote J. B. Fourier (1822), Théorie Analytique de la Chaleur: "L" étude approfondie de la nature est la source la plus féconde des découvertes mathématiques." There is so much evidence in favor of this remark today.

\section{Acknowledgments}

The author thanks his collaborators in the examples of Sec. 5: Ken Downing, Bob Glaeser, Guy Perkins, Bruce Bolt, Reinhold Kaiser, Fady Malik and Ron Vail respectively. This paper is based on talks presented at the 1993 opening ceremonies of the Fields Institute for Research in Mathematical Sciences at the University of Waterloo, Ontario, Canada and at the 1993 Winter Meeting of the Canadian Mathematical Society at Ottawa. The work was partially supported by the NSF Grants DMS-9208683, DMS-9300002, DMS-9625774 and the Office of Naval Research Grant N00014-94-1-0042. Comments of Bob Kass and the referee helped improve the layout and style of the paper.

\section{REFERENCES}

Aki, K. and P. G. Richards, Quantitative Seismology I \& II. Freeman, San Francisco, 1980.

Amato, I., Nobel prizes 1991 Science 254:518-519, 1991. 
Barndorff-Nielsen, O. E. and D. R. Cox, Asymptotic Techniques for Use in Statistics, Chapman and Hall, London, 1989.

Båth, M., Spectral Analysis in Geophysics. Elsevier, Amsterdam, 1994.

Bazin, M. J., P. H. Lucie, and S. Moss de Olivierra, Experimental demonstrations of the mathematical properties of Fourier transforms using diffraction phenomena, Eur. J. Phys. 7: 183-188 (1986).

Benedetto, J. J. and M. W. Frazier, (eds), Wavelets, CRC Press, Boca Raton, 1994.

Berger, J. and R. Wolpert, Estimating the mean function of a Gaussian process and the Stein effect, J. Mult. Anal. 13:401-424, (1983).

Blackman, R. B. and J. W. Tukey, The Measurement of Power Spectra. Dover, New York, 1959.

Bloembergen, N., Nonlinear optics and spectroscopy, Science 215:10571064 (1982).

Bloomfield, P., Fourier Analysis of Time Series: An Introduction, Wiley, New York, 1976.

Blow, D. M. and F. H. C. Crick, The treatment of errors in the isomorphous replacement method, Acta Cryst. 12:794-802 (1959).

Blümich, B., Stochastic nmr spectroscopy, Bull. Magnet. Resonance 7: 5-26 (1985).

Bochner, S., Lectures on Fourier Integrals, Princeton Press, Princeton, 1959.

Bochner, S., Harmonic Analysis and the Theory of Probability, Univ. Calif. Press, Berkeley, 1960.

Bolt, B. A. and J. Butcher, Rayleigh wave dispersion for a single layer on an elastic half space, Australian J. Physics 13:498-504 (1960).

Bolt, B. A., Y. B. Tsai, K. Yeh and M. K. Hsu, Earthquake strong motions recorded at a large near-source array of digital seismographs, Earthquake Eng. Structural Dynam. 10: 561-573 (1982).

Born, M. and Wolf, E. (1964). Principles of Optics. Second Edition. Macmillan, New York.

Bracewell, R. N., The Fourier transform, Scientific Ameri., June: 86-95 (1989).

Brillinger, D. R., A search for a relationship between monthly sunspot numbers and certain climatic series, Bull. Inter. Statist. Inst. 43: 293-306 (1969). 
Brillinger, D. R., Time Series: Data Analysis and Theory, Holt, New York, 1975.

Brillinger, D. R., Asymptotic normality of finite Fourier transforms of stationary generalized processes, J. Mult. Analysis 12:64-71 (1982).

Brillinger, D. R., Some asymptotics of finite Fourier transforms of a stationary p-adic process, J. Comb. Inf. Sys. Sci. 16: 155-169 (1991).

Brillinger, D. R., An application of statistics to seismology: dispersion and modes. In Developments in Time Series Analysis (ed. T. Subba Rao), Chapman and Hall, London, 1993, pp. 331-340.

Brillinger, D. R., Some uses of cumulants in wavelet analysis, Nonpar. Statist. 6: 93-114 (1996).

Brillinger, D. R., K. H. Downing and R. M. Glaeser, Some statistical aspects of low-dose electron imaging of crystals, J. Stat. Planning Inf. 25: 235-259 (1990).

Brillinger, D. R., K. H. Downing, R. M. Glaeser and G. Perkins, Combining noisy images of small crystalline domains in high resolution electron microscopy, J. App. Stat. 16: 165-175 (1989).

Brillinger, D. R. and R. Kaiser, Fourier and likelihood analysis in NMR spectroscopy. In New Directions in Time Series I (eds. D. Brillinger, P. Caines, J. Geweke, E. Parzen, M. Rosenblatt and M. Taqqu), Springer, New York, 1992, pp. 41-64.

Bullen, K. E. and B. A. Bolt, An Introduction to the Theory of Seismology, Cambridge Univ. Press, Cambridge, 1985.

Butzer, P. L. and R. J. Nessel, Fourier Analysis and Approximation, Academic, New York, 1971.

Cartwright, D. E., Tidal analysis - a retrospect in Time Series Methods in Hydrosciences (eds A. H. El-Shaarawi and S. R. Esterby), Elsevier, Amsterdam, 1982, pp. 170-188.

Cooley, J. W. and J. W. Tukey, An algorithm for the machine calculation of complex Fourier series, Math. Comp. 19:297-301 (1965).

Copas, J. B., Regression, prediction and shrinkage, J. Roy. Statist. Soc. B 45: 311-335 (1983).

Cramér, H., On harmonic analysis in certain function spaces, Arkiv Math. Astr. Fysik. 28: 1-7 (1942). 
Dahlhaus, R., Parameter estimation of stationary processes with spectra containing strong peaks in Robust and Nonlinear Time Series Analysis (eds J. Franke, W. Haerdle and D. Martin), Springer, New York, 1984, pp. 50-67.

Dahlhaus, R., Efficient parameter estimation for self-similar processes, Ann. Statist. 17: 1749-1766 (1989).

Daubechies, I., Ten Lectures on Wavelets, SIAM, Philadelphia, 1992.

Diaconnis, P., Group Representations in Probability and Statistics, Institute of Mathematical Statistics, Hayward, 1988.

Diaconnis, P., A generalization of spectral analysis with applications to ranked data, Ann. Statist. 17:949-979 (1989).

Donoho, D. L. and I. M. Johnstone, Wavelets and optimal nonlinear function estimates, Tech. Report 281, Statistics Dept. Univ. California, Berkeley, 1990.

Donoho, D. L., I. M. Johnstone, G. Kerkyacharian and D. Picard. Wavelet shrinkage: asymptopia? J. Roy. Statist. Soc. B 57: 301-369 (1995).

Dzhaparidze, K., Parameter Estimation and Hypothesis Testing in Spectral Analysis of Stationary Time Series, Springer, New York, 1986.

Efron, B. and C. Morris, Stein's paradox in statistics, Scientific Amer. 236: 119-127 (1977).

Fan, J., Deconvolution with supersmooth distributions, Canadian J. Statist. 20: 155-169 (1992).

Feuerverger, A., Efficiency in time series, Canadian J. Statist. 18: 155-162. (1990).

Feigin, P. D. and C. R. Heathcote, The empirical characteristic function and the Cramér-von Mises statistic, Sankhya A 38:309-325 (1976).

Fine, N. J., On Walsh functions, Trans. Amer. Math. Soc. 65:372-414 (1949).

Fox, R. and M. S. Taqqu, Large-sample properties of parameter estimates for strongly dependent stationary time series, Ann. Statist. 14:517-532 (1986).

Freedman, D. and D. Lane, The empirical distribution of the Fourier coefficients of a sequence of independent, identically distributed long-tailed random variables, Z. Wahrschein. Ver. Geb. 55:123-132 (1981). 
Glaeser, R. M., Electron crystallography of biological macromolecules, Ann. Rev. Phys. Chem. 36: 243-275 (1985)

Good, I. J., The interaction algorithm and practical Fourier series, J. Roy. Statist. Soc. B 20:361-372 (1958).

Good, I. J., Weighted covariance for detecting the direction of a Gaussian source in Time Series Analysis (ed. M. Rosenblatt), Wiley, New York, 1963, pp. 447-470.

Goodman, J. W., Introduction to Fourier Optics, McGraw-Hill, San Francisco, 1968.

Gorgui-Naguib, R. N., p-adic transforms in digital signal processing in Mathematics in Signal Processing II (ed. J. G. McWirter), Clarendon, Oxford, 1990, pp. 43-53.

Hall, P. and P. Patil, On wavelet methods for estimating smooth functions, Bernoulli 1 (1995), pp. 41-58.

Halvorson, C., A. Hays, B. Kraabel, R. Wu, F. Wudl and A. J. Heeger, A 160-femtosecond optical image processor based on a conjugated polymer, Science 265: 1215-1216 (1994).

Hannan, E. J., Aliasing. Tech. Report 25, Statistics Dept., Johns Hopkins University, 1965.

Hannan, E. J., Group Representations and Applied Probability, Methuen, London, 1966.

Hannan, E. J., Fourier methods and random processes, Bull. Internat. Statist. Inst. 42(1): 475-496 (1969).

Hannan, E. J., Spectra changing over narrow bands in Statistical Models and Turbulence (eds M. Rosenblatt and C. Van Atta), Springer, New York, 1972, pp. 460-469.

Hannan, E. J. and P. J. Thomson, Spectral inference over narrow bands, $J$. Appl. Prob. 8: 157-169 (1971).

Hayward, S. B. and R. M. Stroud, Projected purple membrane determined to $3.7 \AA$ resolution by low temperature electron microscopy, J. Molec. Biol. 151: 491-517 (1981).

Henderson, R., J. M. Baldwin, K. H. Downing, J. Lepault, and F. Zemlin, Structure of purple membrane from Halobacterium halobium, Ultramicroscopy 19: 147-178 (1986). 
Henderson, R., J. M. Baldwin, T. A. Ceska, F. Zemlin, E. Beckmann and K. H. Downing, Model for the structure of bacteriorhodopsin based on high-resolution electron cryo-microscopy, J. Mol. Biol.213: 899-929(1990).

Hennel, J. W. and J. Klinowski, Fundamentals of Nuclear Magnetic Resonance, Wiley, New York, 1993.

Hewitt, E. and K. A. Ross, Abstract Harmonic Analysis I\&II, Academic, New York, 1963.

Higgins, J. R., Five short stories about the cardinal series, Bull. Amer. Math. Soc. 12: 45-89 (1985).

Hirsch, M. W., The dynamical systems approach to differential equations, Bull. Amer. Math. Soc. 11:1-64 (1984).

Hochstadt, H., Differential Equations, A Modern Approach, Holt-Rinehart, New York, 1964.

Hovmoller, S., Structure analysis by crystallographic image processingHommage à Jean Baptiste Joseph Fourier (1768-1830), Microsc. Microanal. Microstruct. 1: 423-431 (1990).

Ihaka, R., Statistical aspects of earthquake source parameter estimation in the presence of signal generated noise, Commun. Statist. A 22:14251440 (1993).

Katz, B. and R. Miledi, Further observations on acetylcholine noise, Nature 232: 124-126 (1971).

Katznelson, Y., An Introduction to Harmonic Analysis, Dover, New York, 1976.

Kim, P. and G. R. Chapman, Group action on a lattice and an application to time series, J. Stat. Planning Inf. 34: 183-195 (1993).

King, N., An alternative for the linear regression equation when the predictor variable is uncontrolled and the sample size is small, J. Amer. Statist. Assoc. 67:217-219 (1972).

Korner, T. W., Fourier Analysis. Cambridge Univ. Press, Cambridge, 1989.

Lanczos, C., Discourse on Fourier Series, Hafner, New York, 1966.

Leonov, V. P. and A. N. Shiryaev, Some problems in the spectral theory of higher moments, Theory Prob. Appl. 5:460-464 (1960). 
Lillestol, J., Improved estimates for multivariate complex normal regression with application to analysis of linear time-invariant relationships. $J$. Mult. Anal. 7: 512-524 (1977).

Loomis, L., An Introduction to Abstract Harmonic Analysis, Van Nostrand, New York, 1953.

Malik, F., D. R. Brillinger, and R. D. Vale, High resolution tracking of microtubule motility driven by a single kinesin motor, Proc. Natl. Acad. Sci. USA 91, pp. 4584-4588 (1994).

Michaelson, A. A., On the application of interference methods to spectroscopic methods-I. Phil. Mag. 33: 338-346 (1891a).

Michaelson, A. A., On the application of interference methods to spectroscopic methods-II. Phil. Mag. 34:280-299 (1891b).

Moloney, J. V. and A. C. Newell, Nonlinear optics. Tech. Report 574. IMA, University of Minnesota, Minneapolis, 1989.

Ott, J. and R. A. Kronmall, Some classification procedures for multivariate binary data using orthogonal functions, J. Amer. Statist. Assoc. 71:391399 (1976).

Picinbono, M. B., Tendance vers le caractère gaussien par filtrage sélectif, Comptes Rendus Acad. Sci., 1174-1176 (1960).

Priestley, M. B., Evolutionary spectra and non-stationary processes, J. Roy. Statist. Soc. B 27:204-237 (1965).

Richards, F. S. G., A method of maximum-likelihood estimation, J. Roy. Statist. Soc. B 23:469-475 (1961).

Rockmore, D., Fast Fourier analysis for abelian group extensions, $A d v$. Appl. Math. 11: 164-204 (1990).

Rosenberg, J. R., A. M. Amjad, P. Breeze, D. R. Brillinger and D. M. Haliday, The Fourier approach to the identification of functional coupling between neuronal spike trains, Prog. Biophys. Molec. Biol. 53: 1-31 (1989).

Rosenblatt, M., Some comments on narrow band-pass filters, Quart. Appl. Math. 18: 387-394 (1961).

Rosenblatt, M., Probability limit theorems and some questions in fluid mechanics in Statistical Models and Turbulence (eds M. Rosenblatt and C. Van Atta), Springer, New York, 1972, pp. 27-40. 
Rosenblatt, M., Limit theorems for Fourier transforms of functionals of Gaussian sequences, Z. Wahrsch. Verw. Gebiete. 55: 123-132 (1981).

Rudin, W., Fourier Analysis on Groups, Wiley, New York, 1962.

Ruelle, D., Chaotic Evolution and Strange Attractors, Cambridge Univ. Press, Cambridge, 1989.

Saleh, A. K., Contributions to Preliminary Test and Shrinkage Estimation, Department of Mathematics and Statistics, Carleton University, Canada, 1992.

Shao, M. and C. L. Nikias, Signal processing with fractional lower order moments: stable processes and their applications, Proc. IEEE 81:9861010 (1993).

Shankar, P. M., S. N. Gupta, and H. M. Gupta, Applications of coherent optics and holography in biomedical engineering, IEEE Trans. Biomed. Eng. BME-29: 8-15 (1982).

Slutsky, E., Alcuni applicazioni di coefficienti di Fourier al analizo di sequenze eventuali coherenti stazionari, Giorn. d. Instituto Italiano degli Atuari 5: 435-482 (1934).

Smith, K. T., The uncertainty principle on groups, SIAM J. Appl. Math. 50: 876-882 (1990).

Stein, C., Inadmissibility of the usual estimator for the mean of a multivariate normal distribution in Proc. Third Berk. Symp. Math. Statist. Prob. Vol. 1, Univ. Calif. Press, Berkeley, 1955, pp. 197-206.

Stoffer, D. S., Walsh-Fourier analysis and its statistical applications, $J$. Amer. Statist. Assoc. 86:481-482 (1991).

Strang, G., Wavelet transforms versus Fourier transforms, Bull. Amer. Math. Soc. 28: 288-305 (1993).

Strichartz, R. S., How to make wavelets, Amer. Math. Monthly 100: 539-556 (1990).

Tarter, M. E. and M. D. Lock, Model-free Curve Estimation, Chapman and Hall, New York, 1993.

Terras, A., Harmonic Analysis on Symmetric Spaces and Applications I \& II, Springer, New York, 1988.

Thompson, J. R., Some shrinkage techniques for estimating the mean, $J$. Amer. Statist. Assoc. 63: 113-122 (1968). 
Timan, A. F., Theory of Approximation of Functions of a Real Variable, Pergamon, Oxford, 1963.

Tukey, J. W., An introduction to the frequency analysis of time series in The Collected Works of John W. Tukey I (1984). (ed. D. R. Brillinger), Wadsworth, Pacific Grove, 1963, pp. 503-650.

Tukey, J. W., Equalization and pulse shaping techniques applied to determination of initial sense of Rayleigh waves in The Collected Works of John W. Tukey I (1984). (ed. D.R. Brillinger), Wadsworth, Pacific Grove, 1959, pp. 309-358.

Tukey, J. W., Introduction to the dilemmas and difficulties of regression. Unpublished, 1979.

Walter, G. G., Approximation of the delta function by wavelets, J. Approx. Theory 71: 329-343 (1992).

Walter, G. G. (1994). Wavelets and Other Orthogonal Systems with Applications. CRC Press, Boca Raton.

Wenk H. R., K. H. Downing, M. S. Hu and M. A. Okeefe, 3D structure determination from electron-microscope images-electron crystallography of staurolite, Acta Crystallographica A1, 48:700-716 (1992).

Whittle, P., Estimation and information in time series analysis, Skand. Aktuar. 35: 48-60 (1952).

Whittle, P., Discussion of C. M. Stein "Confidence sets for the mean of a multivariate normal distribution", J. Roy. Statist. Soc. B 24:294 (1962).

Wiener, N., The Fourier Integral and Certain of Its Applications, Dover, New York, 1933.

Yaglom, A. M., Second-order homogeneous random fields in Proc. Fourth Berkeley Symp. Math. Statist. Prob. 2, Univ. Calif. Press, Berkeley, 1961, pp. 593-622.

Yajima, Y., A central limit theorem of Fourier transforms of strongly dependent stationary processes, J. Time Series Analysis 10:375-384 (1989).

Yariv, A., Quantum Electronics, Second Edition, Wiley, New York, 1975. 
Zidek, J., Discussion of Copas (1983), Regression, prediction and shrinkage, pp. 347-48. J. Roy. Statist. Soc. B 45:311-335 (1983).

Zygmund, A., Trigonometric Series, Cambridge Univ. Press, Cambridge, 1968.

\section{APPENDIX}

The estimate presented in the middle panel of Fig. 7 is ordinary least squares. (In many time series situations such estimates are asymptotically efficient.)

The model shown in Eq. (5.16) is linear in $\alpha$ and the $\gamma_{n k}$. It may be written

$$
\mathbf{y}=\mathbf{X} \gamma+\mathbf{Z} \alpha+\varepsilon
$$

taking $\mathbf{Z}=[t-\bar{t}]$ and $\mathbf{X}=\left[X_{t k}\right]$, with $X_{t k}=1$ for $k / 2^{n} \leq t / T<(k+1) / T$ and 0 otherwise. It is seen to have the form of an analysis of covariance model. The least squares estimates may be written

$$
\begin{gathered}
\hat{\alpha}=\left(\mathbf{Z}^{\prime} \mathbf{P Z}\right)^{-1} \mathbf{Z}^{\prime} \mathbf{P y} \\
\hat{\gamma}=\left(\mathbf{X}^{\prime} \mathbf{X}\right)^{-1} \mathbf{X}^{\prime}(\mathbf{y}-\mathbf{Z} \hat{\alpha}) \\
\text { with } \mathbf{P}=\mathbf{I}-\mathbf{X}\left(\mathbf{X}^{\prime} \mathbf{X}\right)^{-1} \mathbf{X}^{\prime} .
\end{gathered}
$$

